\title{
Pain experience profile in children with cancer:prospective analysis of 2216 treatment days in a developing country
}

\begin{abstract}
Background:Cancer in children is a potentially curable disease, particularly in developed countries. Conventional chemotherapy is essentially an integral part of treatment, either alone, or as a part of multimodality therapy including surgery \& radiotherapy. How to deal with pain is an integral part of symptom management in pediatric cancer patients in general, with especially great importance in the context of palliative care in developing countries, in which curability lags behind that of developed countries. Proper recognition of underlying pathophysiology and various causes of pain are so essential for pain management, and for ameliorating suffering in the realm of holistic care for children with cancer. The aim of this study is to address and meticulously analyze the spectrum of pain characteristics in children with cancer at an institutional university cancer center to unravel pain profile in these patients as an experience for a developing country.
\end{abstract}

Patients \& Methods:A hospital based, prospective study was conducted, involving pediatric cancer patients, who presented with pain due to cancer itself or its treatment in the period from 2013 Jul to 2015 Jan. Evaluation of patients' documented pain cycles for pain cause, type, and location \& also for pain treatment characteristics was done.

Results: A total of 286 pain cycles was documented comprising 2216 treatment days (range 3-56 days). Disease-related pain was the most frequent cause of pain in our study. Oral mucosa was the most frequent site for treatment-related pain \& strongly correlated with NHL diagnosis. Leukemia was strongly correlated with "the extremities" as a location of bone pain. Visceral pain was most often associated with lymphomas. Neuropathic pain was the least frequent type of pain, however, associated with higher initial pain intensity scores $\&$ longer pain cycle duration.

Conclusion:Children with cancer in the developing countries still have more disease-related pain than their counterparts in the developed countries. Pain experience in pediatric oncology may indirectly reflect presentations of childhood cancer, and could be a surrogate profile for tumor location, metastatic sites, the degree of treatment intensity, likewise the context of the disease state either at diagnosis, during treatment, or at progression.

Keywords: Pain, Childhood cancer, Somatic pain, Visceral pain, Neuropathic pain, Cancer pain
Volume 4 Issue 6 - 2016

\author{
Montaser A Mohamed,' Ahmed M Morsy,2 \\ Khaled F Riad ${ }^{2}$ \\ 'Department of Anesthesiology, South Egypt Cancer Institute, \\ Egypt \\ ${ }^{2}$ Department of Pediatric Oncology, South Egypt Cancer \\ Institute, Egypt
}

Correspondence: Ahmed Mohammed Morsy, Pediatric Oncology Department, South Egypt Cancer Institute, El-Methak St., Assiut, Egypt, Tel 02/01003314522,

Email ahmedmohammed7829@yahoo.com

Received: April 12, 2016 | Published: May 06, 2016
Abbreviations: ALL, Acute Lymphoblastic Leukemia; COPADM, Cyclophosphamide/Oncovin/Prednisolone/Adriamycin/ Methotrexate; CYVE, CY, ARA-C (Cytarabine) / VE, Etoposide (VP-16 Or Vespid); FAB, French-American-British; FLACC Scale, The Face, Legs, Activity, Cry, Consolability Scale; HDMTX, High Dose Methotrexate; LMB-89 Protocol, Lymphoma Malignant B Protocol; NCI, National Cancer Institute; NHL, Non-Hodgkin's Lymphoma; NSAIDS, Non-Steroidal Anti-Inflammatory Drugs; WBCs, White Blood Cells; WHO, World Health Organization

\section{Introduction}

Cancer is a significant cause of persistent pain in children. Its treatment is often complex and requires holistic approach. ${ }^{1} \mathrm{~A}$ significant barrier to pain management in children is that research and development of evidence-based practice guidelines in pediatrics lags behind that in adults. ${ }^{2}$

Pediatric pain can be divided into three major categories, i.e.:somatic, visceral and neuropathic. Somatic pain is caused by tissue injury or inflammation. When involving skin and superficial structures, somatic pain is sharp and well-localized. Visceral pain is caused by inflammation or injury of internal organs (viscera), usually poorly localized or referred to distant locations. Finally, neuropathic pain is caused by injury, inflammation, or dysfunction of the peripheral or central nervous systems. ${ }^{3}$ Pain experienced by children with cancer may be caused by various entities, including the disease itself (e.g., tumor invasion of bone, viscera, peripheral or central nervous system, or compression of the spinal cord), treatment (e.g., mucositis, radiation-induced dermatitis, drug-induced neuropathy), or procedures (e.g., Venipuncture, lumbar puncture, bone marrow aspiration or biopsy, postoperative pain). ${ }^{2}$

World Health Organization (WHO) has proposed a useful approach to drug selection for acute and chronic pain states, which has become known as the "analgesic ladder". The WHO guidelines previously provided a three-step approach to cancer-related pain in children and adults. ${ }^{4,5}$ The WHO ladder states that non-opioids (paracetamol \& NSAIDs) should be administered first, followed by weak opioid 
(Codeine) and then, if required, strong opioids (Morphine). ${ }^{6}$ The current WHO pain guidelines recommend the first step for mild pain and the second step for moderate to severe pain. ${ }^{7}$ For mild pain, paracetamol and ibuprofen are considered as first options in children older than 3months, while morphine is recommended as the first-line strong opioids for moderate to severe pain.

We aim to gain a deep insight about pain characteristics in children with cancer; exploring its causes, pathophysiologic types; investigating locations of its occurrence in relation to the specific disease entities of childhood cancer in the context of a limited resource country, and comparing it with previously published findings.

\section{Patients \& methods}

\section{Study population}

From Jul 2013 to Jan 2015, pediatric cancer patients attending service at the pediatric oncology department at South Egypt Cancer Institute, whose age from one year to 16 years, presented with pain caused by disease, or caused by anticancer treatment, were eligible to be included in the study. Patients presented with procedural pain, or post-operative pain, were excluded from this study. Informed consent was obtained from all patients' guardians and assent from the child as appropriate, and the treatment was approved by the institutional review board at our center.

\section{Pain assessment}

The study included the documented pain cycles in patients presented with pain, according to the eligibility criteria. A pain cycle was defined as a single patient's uninterrupted documentation. After a complete clinical history \& full clinical examination, qualitative assessment of cause, type and location of pain in each pain cycle was done by well-trained resident physicians at our department to make a reasonable clinical diagnosis of the nature of the pain and to have a differential diagnosis of the causes. Demographic data, disease \& treatment characteristics of involved patients also, were collected. All data were recorded. Imaging and laboratory studies may be used if needed to determine $\&$ evaluate the source of pain.

Quantitative assessment of initial pain intensity scores was done by well-trained nurses under supervision of the resident physician, followed by daily average pain intensity scores measured using a 0-10 scale. Also, the pain cycle duration for each pain cycle was documented in days. The pain severity was categorized as follows:

\section{A. Mild pain: 1 - 3 \\ B. Moderate pain:4 - 7 \\ C. Severe pain: $8-10$}

We used the behavioral FLACC scale ${ }^{8}$ for assessment of pain intensity scores in patients whose age from one to seven years, or who incapable for self-report, while the self-rated 0-10 Wong Baker scale ${ }^{9}$ was used for patients whose age older than seven years up to 16 years.

\section{Pain treatment}

We followed the WHO analgesic ladder guidelines ${ }^{5,7}$ for pain management at our 0000000000000 department. Non opioids drugs used at the $1^{\text {st }}$ step of the ladder were either paracetamol, or Ibuprofen. The dose of paracetamol \& ibuprofen used for analgesia was $(15 \mathrm{mg} /$ $\mathrm{kg} /$ dose $\mathrm{q} 6 \mathrm{~h}) \&(5 \mathrm{mg} / \mathrm{kg} /$ dose $\mathrm{q} 6 \mathrm{~h}$ ) respectively.
At the $2^{\text {nd }}$ step of the ladder, morphine (strong opioids) was administered, in low dose $(0.05 \mathrm{mg} / \mathrm{kg} / \mathrm{dose})$ which may be increased if needed, as a two-step strategy, or as an alternative strategy, tramadol (weak opioids) was used in accordance with the three-step strategy WHO ladder. Fentanyl Patch (strong opioid) was used at the $3^{\text {rd }}$ step, if pain control weren't achieved on tramadol at the $2^{\text {nd }}$ step of the ladder. Tramadol was the only weak opioid used in this study. The dose of tramadol used was ( $1 \mathrm{mg} / \mathrm{kg} /$ dose $\mathrm{q} 8 \mathrm{~h}$ ).

Morphine sulfate was given by intravenous route as a bolus dose (q4-6h). The dose of morphine used was $0.05 \mathrm{mg} / \mathrm{kg} / \mathrm{dose}$. There were no subcutaneous or intramuscular applications.

Transdermal fentanyl patch was applied onto the skin every 72 hours (3 days), following the steps under "Applying the patch", according to instructions included in the product leaflet. As the first patch may take up to a day to take effect after it is applied onto the skin, additional doses of intravenous morphine bolus doses were added as needed to control pain in the $1^{\text {st }}$ treatment day. The choice of the drug type, the route of administration, and pain treatment strategy was carried out according to the convenience and drug availability.

\section{Statistical analysis}

Categorical data were expressed as frequencies or percentages, and the statistical differences were compared using the chi-square test. Fisher's exact test was used, where appropriate. Measurement data were expressed as the "mean \pm SD", or as the "median \& inter quartile range", and the statistical differences between groups were compared using the "Mann Whitney U test" or "Kruskal Wallis test" among independent-samples.

All statistical analyses were performed using the Statistical Package for Social Sciences software, SPSS, version 20.0 (SPSS Inc., Chicago, Illinois, USA). Both descriptive and inferential statistical methods were used. For all statistical tests, the threshold for statistical significance (two-tailed) was set to ( $\mathrm{p}$ value $<0.05$ ).

\section{Results}

\section{Patients' characteristics}

The study included 112 patients and their median age was 6 years (range:1-16 years). Sixty-four patients $(57.1 \%)$ were males and 48 patients $(42.9 \%)$ were females. The most frequently occurring diagnosis in the study participants was ALL in (30.4\%), followed by NHL in $(23.2 \%)$, and neuroblastoma in $(17 \%)$ of patients.

A significant difference was observed in the distribution of the age of patients, according to tumor diagnosis ( $p$ value $<0.0005$ ), while no difference was found due to disease state whether disease at diagnosis, at relapse or during treatment $(\mathrm{p}$ value $=0.114)$ (Figure $1 \& 2)$.

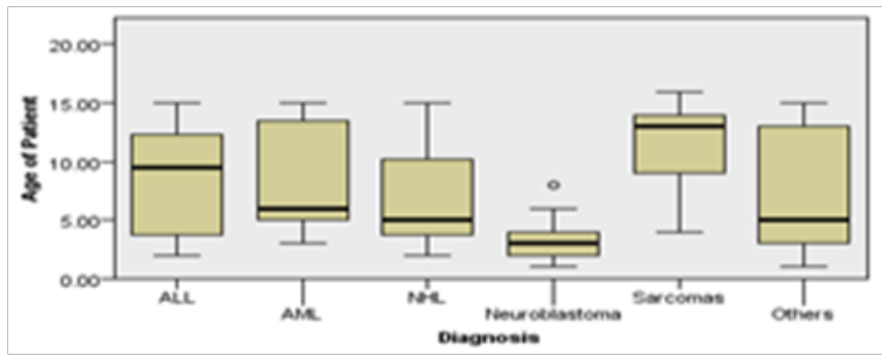

Figure I Distribution by Age of Patients according to Tumor Diagnosis. 


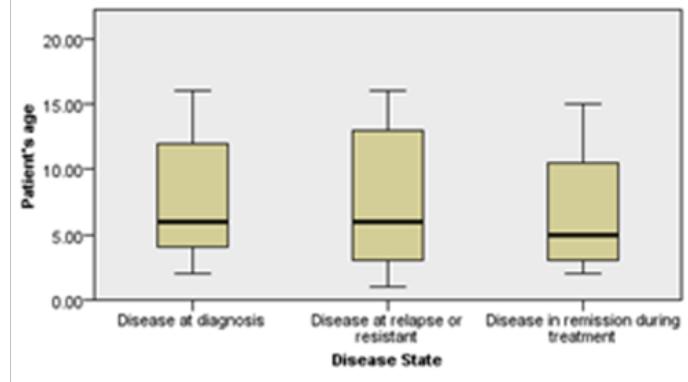

Figure 2 Distribution by Age of Patients according to Disease State.

\section{Causes \& types}

During 18 months duration, we documented 286 pain cycles comprising a total of 2216 treatment days, with median pain cycle duration 6 days (range 3-56 d).Of the documented pain cycles, "disease-related pain" was the most frequent, constituting $(67.1 \%)$, followed by "treatment-related pain" in $(23.8 \%)$, and "inflammation" in $(9.1 \%)$ of pain cycles. "Somatic" type of pain was the most frequent type in the documented pain cycles constituting (77.6\%), followed by "visceral" pain in $(15.4 \%)$, \&"neuropathic" pain in $(7.0 \%)$ of pain cycles. Pain cause and type according to tumor diagnosis were shown in (Figure $3 \& 4$ ).
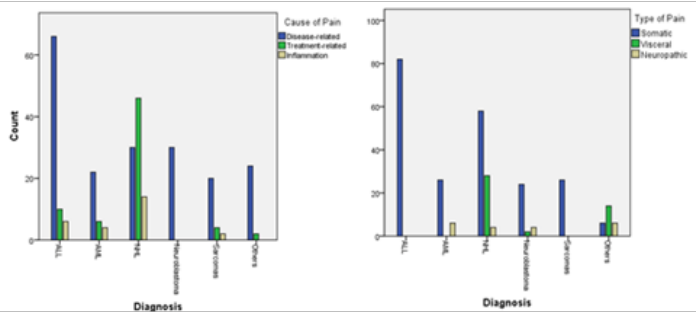

Figure 3 \& 4 Pain Cause \& Type according to Tumor Diagnosis.

Significant differences between various pain characteristics (cause, type \& location) were found across the main diagnostic categories (leukemias, lymphomas \& solid tumors) as shown in (Table 1).

\section{Pain location}

According to pain location, the most frequently occurring sites of pain were "the extremities" which constituted (30.8\%), followed by "oral mucosa" in $(24.5 \%)$, and then by "head \& neck not oral mucosa" location in (16.1\%) of documented pain cycles. Pain location according to tumor diagnosis was shown in (Figure 5-8).

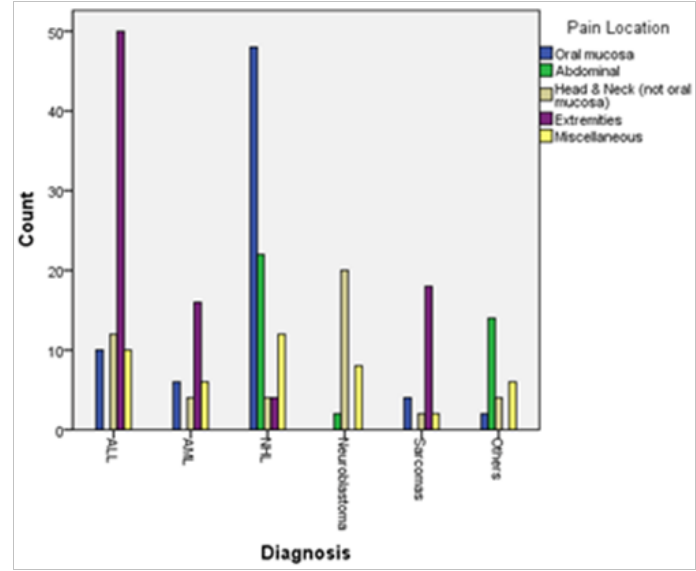

Figure 5 Pain Location according to Tumor Diagnosis.

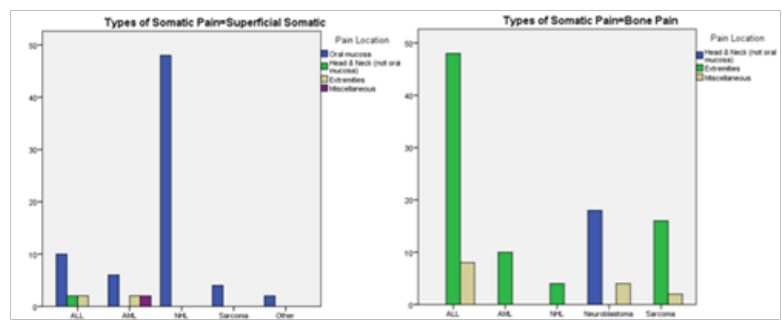

Figure 6 \& 7 Pain Location according to Tumor Diagnosis in selected subcategories of Somatic Pain (Superficial Somatic \& Bone Pain Types).

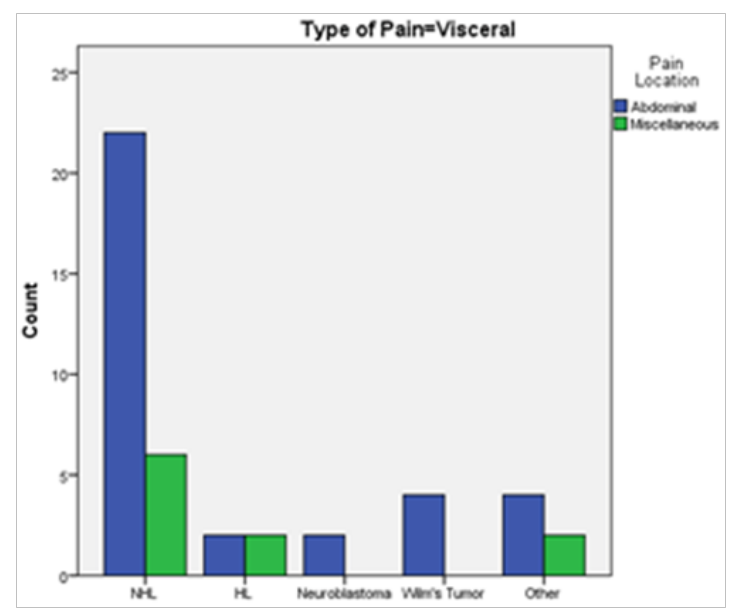

Figure 8 Pain Location according to Tumor Diagnosisin (Visceral Pain Category).

\section{Pain intensity \& pain cycle duration}

No differences were found in the distribution of initial pain intensity scores $(\mathrm{p}$ value $=0.289)$, or pain cycle duration in days $(\mathrm{p}$ value $=0.780)$ due to gender of patients (Figure $9 \& 10$ ).

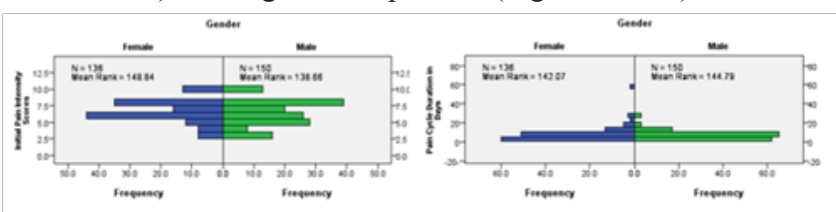

Figure 9 \& 10 Initial Pain Intensity Scores \& Pain Cycle Duration by Gender.

After exclusion of four pain cycles whose patients were diagnosed with brain tumors, solid tumors had been found to be associated with higher initial pain intensity scores than hematological malignancies ( $p$ value $<0.0005)$, but no difference was found between the two groups in the pain cycle duration ( $\mathrm{p}$ value $=0.571$ ) (Figure $11 \& 12$ ).
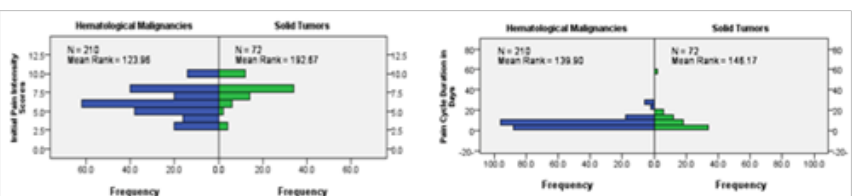

Figure I I \& I 2 Initial Pain Intensity Scores \& Pain Cycle Duration by Disease Category.

Resistant tumors or those at relapse had been found to be associated with higher initial pain intensity scores ( $p$ value $<0.0005) \&$ longer pain cycle duration ( $\mathrm{p}$ value $<0.0005)$ than those at diagnosis or in remission under treatment (Figure $13 \& 14$ ). 


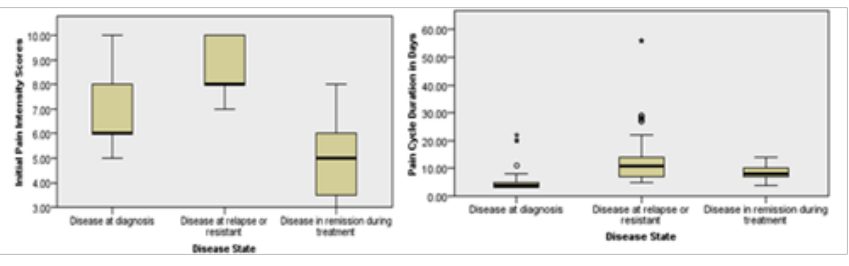

Figure I 3 \& I 4 Initial Pain Intensity Scores \& Pain Cycle Duration by Disease State.

The median of initial pain intensity scores in the treatment-related pain group was "four", which was lower than that of the disease-related pain group, whose median score was "seven" $(p<0.0005)$. On the other hand, the median of pain cycle duration in the treatment-related pain group was "eight days", which was longer than that of the disease-related pain group, whose median duration was "five days" ( $p$ value $<0.0005$ ) (Figure $15 \& 16$ ).
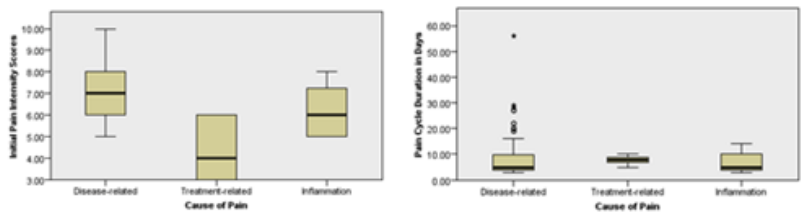

Figure I 5 \& 16 Initial Pain Intensity Scores \& Pain Cycle Duration by Cause of Pain.

Neuropathic type of pain was found to be associated with higher pain intensity scores $(\mathrm{p}$ value $<0.0005)$ and longer pain cycle durations ( $p$ value $<0.0005)$ than somatic or visceral types of pain (Figure 17 - 20).
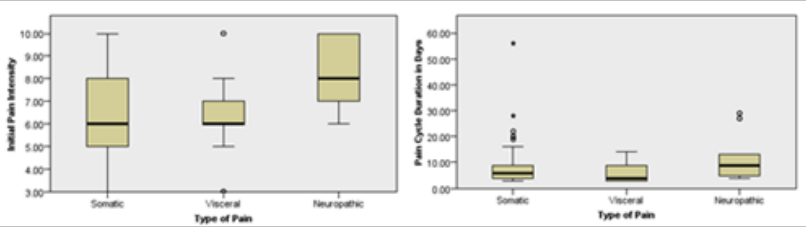

Figure 17 \& 18 Initial Pain Intensity Scores \& Pain Cycle Duration by Cause of Pain.

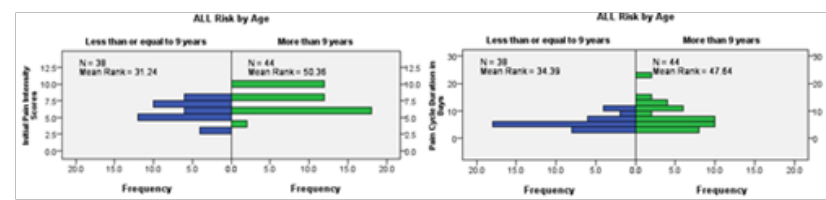

Figure 19 \& 20 Initial Pain Intensity Scores \& Pain Cycle Duration in ALL patients according to (Risk Stratification by Age).

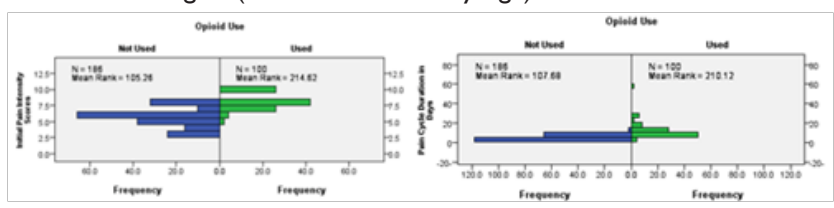

Figure 2 I \& 22 Initial Pain Intensity Scores \& Pain Cycle Duration according to whether Opioid Used or not used.

\section{Pain treatment characteristics}

Non-opioid drug was used in about $45.5 \%$ of all documented treatment days, while weak opioid \& strong opioid drugs were used in $20.2 \%$ \& $34.3 \%$ of all documented treatment days respectively. One hundred \& eighty-sex pain cycles $(65 \%)$ were treated within one WHO ladder step. Seventy-eight pain cycles $(27.3 \%)$ were treated within two WHO ladder steps, i.e. WHO ladder treatment steps were changed once, and 22 pain cycles $(7.7 \%)$ were treated within three WHO ladder steps, i.e. WHO ladder treatment steps were changed twice.

Pain cycles, where opioid drugs were used, were associated with higher initial pain intensity scores and longer pain cycle duration than those where opioid drugs weren't used. By further analysis of the group where opioid drugs were used, no difference was found in the opioid drugs used, whether weak or strong, or the WHO ladder strategy used, whether two-step or three-step strategy, across the main diagnostic categories (Leukemias, lymphomas \& solid tumors) (Figure $21 \& 22$ ) (Table 2).

\section{Discussion}

Differently from the adult patients, in pediatric age it is more difficult to assess and treat efficaciously the pain and often this symptom is undertreated or not treated. In children, selection of appropriate pain assessment tools should consider age, cognitive level and the presence of eventual disability, type of pain and the situation in which it is occurring. ${ }^{10}$ In the present study we used the behavioral FLACC scale ${ }^{8}$ for pain assessment in children aged from one year to seven years, while the self-rated 0-10 Wong Baker scale ${ }^{9}$ was used in older children up to 16 years.

Our study aimed at exploring pain characteristics in children with cancer with referral to Egypt as an example for a low resource country, comparing our results versus those of developed countries and explaining possible causes for any difference.

By evaluation of the documented pain cycles, we found that "disease-related pain" was the most frequent, constituting (67.1\%), followed by "treatment-related pain" in (23.8\%), and "inflammation" in $(9.1 \%)$.On the contrary, the German study of Zernikow et al. ${ }^{11}$ revealed that treatment related pain was encountered in $(56 \%)$, followed by the tumor in $(21 \%)$, and inflammation in $(13 \%) .{ }^{11}$

With regard to the cause of pain in the main diagnostic categories, we found that "disease-related pain" was most frequently encountered in leukemias, $45.8 \%$ ( 88 out of 192), followed by solid tumors in $36.5 \%$ (70 out of 192), while "treatment-related pain" was most frequently encountered in lymphomas 67.6\% (46 out of 68) (P value $<0.0005$ ) Table 1 . The discrepancy inside the main diagnostic groups in our study, and on the other hand between our findings \& findings of other studies in respect to the cause of pain may be explained on the basis of many factors related to the patient, the disease, the treatment, and the available resources in the context of low income country.

Patient related factors as low educational level \& low income of the family, which possibly have led to lack of awareness, reluctance to seek medical advice, and to attend to hospital on time, that not only would contribute to advanced disease stages at diagnosis but also to poor compliance to treatment and abandonment that would lead to treatment failure \& more disease-related pain.

Disease related factors observed in our sample were; first, patient initial presentation with late advanced stage disease, particularly seen in solid tumors (e.g., neuroblastoma \& sarcomas) whose treatment requires multimodality therapy for both local control, side by side, systemic control of micro-metastases that couldn't be achieved in late stages of the disease; second, biologically inherently high risk groups were overrepresented in our sample, as higher ages of acute lymphoblastic leukemia (Median age $=10$ years), so about $53.7 \%$ of pain cycles were for NCI high risk ALL (Age > 9 years), despite 
not including other risk criteria as total WBCs. We further explored the difference between the two groups, whether ( $>9$ or $<=9$ years), we found statistically significant higher initial intensity pain scores and longer pain cycle duration in the high risk group (Age $>9$ years) (Figure $19 \& 20$ ). According to the best of our knowledge, prognostic risk criteria haven't been used before to investigate pain experience burden in children with cancer.

Treatment related factors due to associated comorbidities as malnutrition \& infectious hepatitis may drive us to modify treatment and to give less intensified therapy to reduce the potential toxicities of treatment in these vulnerable patients. Other administrative factors as timely lack of essential chemotherapeutic agents may contribute to less adherence to treatment protocols and possible treatment failure \& more cases with relapse and eventually more disease related pain e.g., as recurrent lack of 1-asparginase, which is considered an essential integral part of induction therapy in ALL and no known alternative drug could replace its shortage. Finally, financial resources are also limited, albeit some voluntary donations may help.

Table I Various Pain Characteristics (Cause, Type \& Location) according to the main diagnostic categories (Leukemias, Lymphomas \& Solid tumors)

\begin{tabular}{|c|c|c|c|c|c|c|}
\hline \multirow{2}{*}{\multicolumn{2}{|c|}{ Pain characteristics }} & \multicolumn{3}{|c|}{ Diagnostic category } & \multirow{2}{*}{$\begin{array}{l}\text { Total } \\
286 \text { (100\%) }\end{array}$} & \multirow{2}{*}{ P Value } \\
\hline & & Leukemias & Lymphomas & Solid tumors & & \\
\hline \multirow{4}{*}{ Cause of Pain } & Disease-related & $\begin{array}{l}88 \\
77.20 \%\end{array}$ & $\begin{array}{l}34 \\
36.20 \%\end{array}$ & $\begin{array}{l}70 \\
89.70 \%\end{array}$ & $\begin{array}{l}192 \\
67.10 \%\end{array}$ & \multirow{3}{*}{0.0005} \\
\hline & Treatment-related & $\begin{array}{l}16 \\
14.00 \%\end{array}$ & $\begin{array}{l}46 \\
48.90 \%\end{array}$ & $\begin{array}{l}6 \\
7.70 \%\end{array}$ & $\begin{array}{l}68 \\
23.80 \%\end{array}$ & \\
\hline & Inflammation & $\begin{array}{l}10 \\
880 \%\end{array}$ & 14 & $260 \%$ & 26 & \\
\hline & & $\begin{array}{l}8.80 \% \\
108\end{array}$ & $\begin{array}{l}14.90 \% \\
58\end{array}$ & $\begin{array}{l}2.60 \% \\
56\end{array}$ & $\begin{array}{l}9.10 \% \\
222\end{array}$ & \multirow{4}{*}{0.0005} \\
\hline \multirow{3}{*}{ Type of Pain } & Somatic & $94.70 \%$ & $61.70 \%$ & $71.80 \%$ & $77.60 \%$ & \\
\hline & Visceral & $\begin{array}{l}0 \\
0.00 \%\end{array}$ & $\begin{array}{l}32 \\
34.00 \%\end{array}$ & $\begin{array}{l}12 \\
15.40 \%\end{array}$ & $\begin{array}{l}44 \\
15.40 \%\end{array}$ & \\
\hline & Neuropathic & $\begin{array}{l}6 \\
5.30 \%\end{array}$ & $\begin{array}{l}4 \\
4.30 \%\end{array}$ & $\begin{array}{l}10 \\
12.80 \%\end{array}$ & $\begin{array}{l}20 \\
7.00 \%\end{array}$ & \\
\hline \multirow{6}{*}{ Pain Location } & Oral mucosa & $\begin{array}{l}16 \\
14.00 \%\end{array}$ & $\begin{array}{l}48 \\
51.10 \%\end{array}$ & $\begin{array}{l}6 \\
7.70 \%\end{array}$ & $\begin{array}{l}70 \\
24.50 \%\end{array}$ & \multirow{6}{*}{0.0005} \\
\hline & Abdominal & 0 & 24 & 14 & 38 & \\
\hline & Head \& Neck & 16 & & 26 & & \\
\hline & (not oral mucosa) & $14.00 \%$ & $4.30 \%$ & $33.30 \%$ & $16.10 \%$ & \\
\hline & Extremities & $\begin{array}{l}66 \\
57.90 \%\end{array}$ & $\begin{array}{l}4 \\
4.30 \%\end{array}$ & $23.10 \%$ & $30.80 \%$ & \\
\hline & Miscellaneous & 16 & $\begin{array}{l}14 \\
14.90 \%\end{array}$ & $\begin{array}{l}14 \\
17.90 \%\end{array}$ & $\begin{array}{l}44 \\
15.40 \%\end{array}$ & \\
\hline
\end{tabular}

Table 2 Opioid Drugs Used \&WHO Ladder Strategy according to Diagnostic Category

\begin{tabular}{|c|c|c|c|c|c|c|}
\hline \multirow{2}{*}{\multicolumn{2}{|c|}{ Opioid drugs \& WHO ladder strategy }} & \multicolumn{3}{|c|}{ Diagnostic category } & \multirow{2}{*}{$\begin{array}{l}\text {-Total I00 } \\
-100 \%\end{array}$} & \multirow[b]{2}{*}{$P$ value } \\
\hline & & $\begin{array}{l}\text { Leukemias } \\
50(100 \%)\end{array}$ & $\begin{array}{l}\text { Lymphomas } \\
10(100 \%)\end{array}$ & $\begin{array}{l}\text { Solid tumors } \\
40(100 \%)\end{array}$ & & \\
\hline \multirow{4}{*}{$\begin{array}{l}\text { Weak Opioid Vs. Strong } \\
\text { Opioid }\end{array}$} & \multirow{2}{*}{ Weak Opioid } & 14 & 4 & 20 & 38 & \multirow{4}{*}{0.101} \\
\hline & & $28.00 \%$ & $40.00 \%$ & $50.00 \%$ & $38.00 \%$ & \\
\hline & \multirow{2}{*}{ Strong Opioid } & 36 & 6 & 20 & 62 & \\
\hline & & $72.00 \%$ & $60.00 \%$ & $50.00 \%$ & $62.00 \%$ & \\
\hline \multirow{6}{*}{ Opioid Drug Used } & \multirow{2}{*}{ Tramadol Only } & 14 & 4 & 20 & 38 & \multirow{6}{*}{0.306} \\
\hline & & $28.00 \%$ & $40.00 \%$ & $50.00 \%$ & $38.00 \%$ & \\
\hline & & 24 & 4 & 12 & 40 & \\
\hline & Morphine & $48.00 \%$ & $40.00 \%$ & $30.00 \%$ & $40.00 \%$ & \\
\hline & Tramadol \& Fentanyl & 12 & 2 & 8 & 22 & \\
\hline & Patch & $24.00 \%$ & $20.00 \%$ & $20.00 \%$ & $22.00 \%$ & \\
\hline \multirow{4}{*}{$\begin{array}{l}\text { WHO Ladder } \\
\text { 2-Step Vs. 3-Step }\end{array}$} & '2 Step Strategy WHO & 26 & 6 & 28 & 60 & \multirow{4}{*}{0.223} \\
\hline & Ladder' & $52.00 \%$ & $60.00 \%$ & $70.00 \%$ & $60.00 \%$ & \\
\hline & '3 Step Strategy WHO & 24 & 4 & 12 & 40 & \\
\hline & Ladder' & $48.00 \%$ & $40.00 \%$ & $30.00 \%$ & $40.00 \%$ & \\
\hline
\end{tabular}

Mucositis is defined as inflammatory and/or ulcerative lesions of the oral and/or gastrointestinal tract. One of the major causes of mucositis is high-dose cancer therapy. ${ }^{12}$ Acute oral pain is a classic symptom of oral mucositis. ${ }^{13}$ Oral pain associated with mucositis may be categorized as superficial somatic pain ${ }^{14}$ Typically, oral symptoms develop 5 to 8 days after the administration of chemotherapy and last approximately 7 to 14 days, ${ }^{15}$ a finding that was comparable to the median pain cycle duration in treatment-related pain group in our study, which was 8 days (range 5-10) (Figure 16), and this may be explained on the basis of biologically inherent healing time $\&$ turnover renewal capacity of the oral mucosal epithelium. On the other hand, median pain cycle duration of disease-related pain was 5 days (range
3-56), with a highly diverse range that reflect for the most part the variability of pain cycle duration at initial diagnosis whose median was 4 days, against it at disease relapse or progression whose median was 11 days (Figure 14).

Chemotherapy with methotrexate and other anti metabolites leads to a $20-60 \%$ rate of alimentary tract mucositis according to the drug's given dose per cycle. ${ }^{12}$ The toxicity of each drug depends on its dosage and the exposure time as well as its intrinsic properties. ${ }^{16}$ Most anticancer drugs reach the mucous membrane through the blood, but some, such as methotrexate and etoposide, can be found in the salivary fluid, thus having a direct effect on epithelium. ${ }^{15}$ 
In the current study, oral mucosal pain was strongly correlated with NHL diagnosis (Pearson Contingency Coefficient; $r=.716$, $\mathrm{P}<.0005)$ (Figure $5 \& 6$ ), where 46 out of $90(51.1 \%)$ NHL pain cycles were "treatment-related" due to oral mucositis, a finding very close to $(56 \%)$ that reported by Zernikow et al. ${ }^{11}$ regarding treatment-related pain. This may be explained by administration of intensive chemotherapy in the form of partially modified LMB 89 Protocol that includes (COPADM 1 \& 2) during induction, followed by a consolidation phase (2 cycles of CYVE) after proper staging according to the FAB staging system for B cell lymphoma. Similarly, our finding was comparable with that of Pourtsidis et al. ${ }^{16}$ who stated that 57 out of $76(75 \%)$ NHL patients treated with FAB LMB 89, 96 protocols, and 2003 modifications have developed oral mucositis post COPADM induction (14 of them post CYVE consolidation, or the $1^{\text {st }}$ maintenance cycle), ${ }^{17}$ however, the lower frequency of mucositis in our study may be explained by chemotherapy modification (reduced doses of HDMTX \& Adriamycin).

With regard to pain pathophysiology in the study, "Somatic" type of pain had been the most frequently occurring type in the documented pain cycles constituting (77.6\%), followed by "visceral" pain in $(15.4 \%), \&$ "neuropathic" pain in $(7.0 \%)$ of pain cycles. Our findings are more or less comparable with those of Niscola et al. ${ }^{18}$ whose study, yet involving adults with hematologic cancer, revealed that, for a total number of 223, pathophysiology was as follows:103 (46\%) deep somatic, deriving from bone in most cases, $44(20 \%)$ superficial somatic (mucositis and cutis derangements), 40 (18\%) visceral, 12 $(5 \%)$ neuropathic, $24(11 \%)$ mixed or due to unknown mechanisms. ${ }^{15}$ Also, our results were similar to those reported by Abdel-Hadi et al. ${ }^{19}$ whose study, which was involving pediatric cancer patients revealed that, out of 133 pain cycles, 'Somatic' pain was the most frequent type, constituting (77.4\%), followed by 'Visceral' type in (16.5\%), and 'Neuropathic' type in $(6.0 \%)$ of pain cycles. ${ }^{19}$

Moreover, we observed that somatic pain was most frequently associated with leukemias, while visceral pain was most frequently associated with lymphomas (P value $<0.0005$ ) (Figure $4,8 \&$ Table 1). Another study, dealing with the pain experienced by hospitalized hematologic malignancy adult patients, visceral pain was mostly observed in non-Hodgkin lymphomas. ${ }^{20,21}$

Furthermore, deep somatic pain, deriving from bone in most cases, as those of Niscola et al. ${ }^{15}$ study, we found that bone pain, constituted the majority $76.4 \%$ (110 out of 144) of deep somatic pain. Out of 110 bone pain cycles, leukemias were the most frequent, $60 \%(66$ out of 110), followed by neuroblastoma in $20 \%$ (22 out of 110), and sarcomas in $16.4 \%$ (18 out of 110) (Figure 7).

In addition, we observed that 29 out of $82(35.4 \%)$ pain cycles of ALL patients were caused by bone pain at the initial diagnosis. This finding was comparable to that reported by Jonsson et al. ${ }^{22}$ where bone pain was found in 117 out of $296(40 \%)$ of patients at the onset of pediatric ALL $\left.{ }^{22}\right]$. Starting treatment was reported to ameliorate disease-related pain in a median of 3 days in a study of patients with leukemia. ${ }^{23}$ This finding was confirmed in our study where a median of 4 days was required for leukemia cancer-related pain to be resolved after institution of treatment.

Although neuropathic pain was the least frequent type in the current study; however, it has been associated with higher initial intensity pain scores \& longer pain cycle duration. Generally speaking, neuropathic pain is much less frequent in children with cancer than those of adults, but still has a greater burden in pain management than other types, likewise what reported by Anghelescu, et al. in their study that $15 \%$ patients referred to their pediatric oncology center's pain management pain service had neuropathic pain, and these patients had a significantly greater mean number of pain visits per consultation and significantly more days of pain service follow-up than did other patients.

Regarding pain location, leukemias in particular ALL, and sarcomas specifically bone sarcomas, were found to be strongly correlated with the extremities as sites for bone pain. On the other hand, Neuroblastoma was found to be strongly correlated with the head \& the neck (other than oral mucosa) as a site for bone pain, where bone or intracranial/orbital metastases had occurred (Pearson Contingency Coefficient; $r=.679, \mathrm{P}<.0005$ ) (Figure 7). The Increase of advanced stage neuroblastoma in our study sample, those presented with bone or intracranial/orbital metastases could be partially accounted for by the exclusion of patients whose age less than one year from the study.

Broadly speaking, over representation of high risk disease groups in our study may be explained by either of two possibilities; the first, the patients, those of the higher risk groups would be more liable to develop pain, thus would be more eligible to be included in the study; the second, the prevalence of the higher risk groups of childhood cancer population in our department may be high at the baseline.

We conclude from the above that, Pain experience in pediatric oncology may indirectly reflect presentations of childhood cancer, and could be a surrogate profile for tumor location, metastatic sites, the degree of treatment intensity, likewise the context of the disease state either at diagnosis, during treatment, or at progression.

\section{Limitations}

The study is not without its shortcomings, the major limitation of this study was that it was a hospital-based study, so precluding generalization for a whole spectrum of pediatric cancer population as there may be underrepresentation of terminally-dying children or long-term survivors who may stay at home or present to the outpatient clinic, respectively.

The second limitation was that; although the study was prospective from the time perspective, but it wasn't longitudinal in design, in other words, the patients hadn't been longitudinally followed from the time of diagnosis throughout the time till finishing treatment, so epidemiological inference for whole pediatric population in our department couldn't be achieved.

An additional limitation was underrepresentation of brain tumors in our sample, although they represent a major category of childhood cancer.

\section{Conclusion}

Children with cancer in the developing countries still have more disease-related pain than their counterparts in the developed countries. So the judicious intensification of cancer-directed therapy while maintaining optimal supportive care would lessen the burden of disease-related pain in these countries, but virtually at the expense of the inevitable increase of the burden of treatment-related pain that will warrant more optimization of supportive care $\&$ better control of pain by use of more opioids to maintain the patients long-term survival without compromising their quality of life.

\section{References}

1. Afshan G, Bashir K. Cancer pain in children:a two-step strategy. Anaesth Pain \& Intensive Care. 2004;18(1):106-110. 
2. Ullrich CK, Billett AL. Nathan and Oski's Hematology and Oncology of Infancy and Childhood. Orkin SH, et al, (Eds.), (8th edn), Elsevier Health Sciences, Saunders, Philadelphia, USA. 2014;:2349-2396.

3. Zeltzer LK, EJ Krane. Pediatric Pain Management. Nelson textbook of pediatrics. In:Kliegman RM, et al. Elsevier Health Sciences, USA, 2015;pp. 430-447.

4. WHO. Cancer pain relief. World Health Organization, Geneva, Switzerland, 1986;p. 1-62.

5. WHO. Cancer pain relief and palliative care in children. World Health Organization, Geneva, Switzerland, 1998;p. 1-76.

6. Raphael J, Ahmedzai S, Hester J, et al. Cancer Pain:Part 1:Pathophysiology; Oncological, Pharmacological, and Psychological Treatments:A Perspective from the British Pain Society Endorsed by the UK Association of Palliative Medicine and the Royal College of General Practitioners. Pain Medicine. 2011;11(5):742-764.

7. WHO (2012) WHO guidelines on pharmacological treatment of persisting pain in children with medical Illnesses. World Health Organization, pp. 1-156.

8. Merkel S, Voepel-Lewis T, Malviya S. Pain Assessment in Infants and Young Children:The FLACC Scale:A behavioral tool to measure pain in young children. $A J N$. 2002;102(10):55-58.

9. Chiaretti A, Pierri F, Valentini P, et al. Current practice and recent advances in pediatric pain management. Eur Rev Med Pharmacol Sci. 2003;17(suppl 1):112-126.

10. Wong D, Hockenberry-Eaton M. Wong's essentials of pediatric nursing, Mosby, St Louis, USA.

11. Zernikow B, Smale H, Michel E, et al. Paediatric cancer pain management using the $\mathrm{WHO}$ analgesic ladder-results of a prospective analysis fromn 2265 treatment days during a quality improvement study. Eur J pain. 2006;10(7):587-587.

12. Peterson DE, Bensadoun RJ, Roila F, et al. Management of oral and gastrointestinal mucositis:ESMO Clinical Practice Guidelines. Ann oncol. 2011; 22(suppl 6):vi78-vi84.
13. Brown CG, Wingard J. Clinical consequences of oral mucositis. Semin Oncol Nurs. 2004;20(1):16-21.

14. Niscola P, Romani C, Cartoni C, et al. Epidemiology of pain in hospital haematological setting:an Italian survey. Leuk res. 2008;32(1):197-198.

15. Niscola P, Romani C, Cupelli L, et al. Mucositis in patients with hematologic malignancies:an overview. Haematologica. 2007;92(2):222-231.

16. Niscola P, Scaramucci L, Romani C, et al. Mucositis in the treatment of haematological malignancies. Ann Oncol. 2008;19(Suppl 7):vii141-vii145.

17. Pourtsidis A, Servitzoglou M, Baka M, et al. Pediatric B-cell Non-Hodgkin Lymphoma:21-year Experience with FAB-LMB Protocols in a Single Institute in Greece. Int J Blood Res Disord. 2005;2(2):1-4.

18. Niscola P, Cartoni C, Romani C, et al. Epidemiology, features and outcome of pain in patients with advanced hematological malignancies followed in a home care program:an Italian survey. Ann hematol. 2007;6(9):671-676.

19. Abdel-Hadi S, Ghazaly MM, Montaser MA, et al. Impact of pain management using the WHO analgesic ladder in children with cancer in South Egypt Cancer Institute, Assiut University. SECI Oncology. 2014;2014(1):2-8.

20. Niscola P, Romani C, Scaramucci L, et al. Pain syndromes in the setting of haematopoietic stem cell transplantation for haematological malignancies. Bone Marrow Transplant. 2008;41(9):757-764.

21. Niscola P, Tendas A, Scaramucci L, et al. Pain in malignant hematology. Expert rev hematol. 2011;4(1):81-93.

22. Jonsson OG, Sartain P, Ducore JM, et al. Bone pain as an initial symptom of childhood acute lymphoblastic leukemia:association with nearly normal hematologic indexes. The Journal of pediatrics. 1990;117(2):233-237.

23. Miser AW, McCalla J, Dothage JA, et al. Pain as a presenting symptom in children and young adults with newly diagnosed malignancy. Pain. 1987;29(1):85-90. 